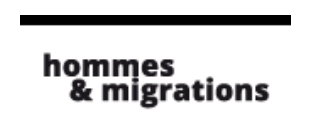

\section{Hommes \& migrations}

Revue française de référence sur les dynamiques

migratoires

$1285 \mid 2010$

L'appel du pied

\title{
Des terrains aux tribunes : sortir le racisme
}

Entretien réalisé par Marie Poinsot avec Carine Bloch

\section{Marie Poinsot et Carine Bloch}

\section{(2) OpenEdition}

1 Journals

\section{Édition électronique}

URL : http://journals.openedition.org/hommesmigrations/1192

DOI : 10.4000/hommesmigrations. 1192

ISSN : 2262-3353

Éditeur

Musée national de l'histoire de l'immigration

\section{Édition imprimée}

Date de publication : 1 mai 2010

Pagination : 148-156

ISSN : 1142-852X

\section{Référence électronique}

Marie Poinsot et Carine Bloch, «Des terrains aux tribunes : sortir le racisme », Hommes \& migrations

[En ligne], 1285 | 2010, mis en ligne le 01 mai 2012, consulté le 30 avril 2019. URL : http://

journals.openedition.org/hommesmigrations/1192; DOI : 10.4000/hommesmigrations.1192 


\section{Des terrains aux tribunes : sortir le racisme}

Entretien réalisé par Marie Poinsot avec Carine Bloch, vice-présidente de la Ligue internationale contre le racisme et l'antisémitisme (Licra), responsable de la commission sport.

En marge des rencontres ou dans les tribunes, les actes racistes, les violences et les discriminations se multiplient. Les stades sont le lieu choisi par certains groupes de supporters pour développer leur activisme

politique. Face à eux, la Licra met en œuvre des partenariats avec les instances dirigeantes du football. L'objectif est de développer des actions tant sur le plan préventif que sur le plan répressif, afin de défendre les victimes et de faire passer un message : dans le sport, 


\section{H\&M : Vous avez travaillé sur les questions de violence et de discrimination liées au sport ?}

Carine Bloch : De 1993 à 2003, j'ai d'abord travaillé dans le secteur du sport. J'ai débuté ma carrière au service des sports de la mairie de Strasbourg (de 1993 à 1997). J'ai remarqué à cette occasion beaucoup de problèmes dans le domaine du sport, mais j'ai constaté également que le sport pouvait être un outil de prévention et de sensibilisation.

La Licra ne travaillait pas sur le sport à l'époque. En mai 1998, juste avant la Coupe du monde, j'ai proposé au comité directeur de la Licra de s'engager sur ces questions, avec un double objectif : utiliser, d'une part, le sport comme outil de prévention et, d'autre part, lutter contre les dérives qu'il y avait dans le sport même, et notamment dans le football.

\section{H\&M : Quel était, en 1998, votre diagnostic concernant les actes de racisme, les violences et les incivilités ?}

C. B.: Malheureusement, le même qu'aujourd'hui. Il y a de l'activisme politique dans des tribunes de football professionnel. Il y a aussi des problèmes de racisme dans le sport amateur, en particulier dans le football. Ce racisme existe dans la société, mais il est exacerbé autour des terrains. Enfin, on remarque des dérives extrémistes, et notamment les pratiques de sectes et d'intégristes qui viennent recruter par le sport dans les quartiers.

La Licra s'intéresse à tous ces problèmes, et aussi au fait qu'il n'y ait pas dans les pôles décisionnels la même diversité que celle que l'on trouve sur les terrains.

En revanche, il faut toujours rappeler que le sport est exemplaire à l'égard de l'égalité des chances, en termes d'accès à la pratique sportive. Au niveau de la pratique loisir, de la pratique de haut niveau ou de la formation, on a une véritable égalité des chances : on départage les sportifs par rapport à leur talent et à leur travail, sans tenir compte de leur origine sociale ou de leur couleur de peau. Il y a un bémol tout de même, au niveau du genre : lorsque l'on compare la place des femmes à celle des hommes, on n'est plus dans un rapport d'égalité.

\section{H\&M : Une femme a-t-elle moins de chance de faire carrière dans le sport ? Rencontre-t-elle plus de difficultés dans son parcours ?}

C. B. : Déjà, il faut dire qu'une fille, on doit aller "la chercher". À l'adolescence, les jeunes filles sont moins demandeuses. À l'opposé, il y a une forte demande des garçons, à laquelle il est difficile de répondre. Certains freins cultuels et culturels face à la pratique féminine du sport interviennent également. Enfin, le sport féminin de haut niveau n'étant pas très valorisé, cela entraîne moins de vocations, 
que chez les garçons. Si on ne met pas en place de politique volontariste par rapport aux filles, elles pratiquent moins. C'est dommage car le sport est un véritable facteur d'émancipation.

Le vivier est moins important, donc les résultats des filles sont moins bons par rapport à ceux des garçons. On a un manque de valorisation du sport et par conséquent des jeunes filles qui le pratiquent. C'est le serpent qui se mord la queue. Il est aussi important_de faire comprendre aux garçons que le sport est également fait pour les filles. Enfin, tant que l'on n'aura pas des femmes nombreuses dans les pôles décisionnels (au niveau des dirigeants, arbitres, entraîneurs, etc.), on n'aura personne pour se focaliser sur cette question.

\section{H\&M : En 1998, lorsque vous avez commencé votre travail à la Licra, comment était identifié le problème du racisme et des discriminations dans les médias ?}

C. B. : Le racisme dans le football ne s'est pas arrêté en 1998. Je dirais même que la Coupe du monde et le discours tenu sur l'équipe "Black Blanc Beur" nous a desservis et a compliqué la tâche dans nos relations avec les instances dirigeantes du football. On voulait surfer sur cette image positive, sans vouloir voir les dérives. Du coup, la question du racisme dans le football professionnel et amateur est restée un sujet tabou, et ce jusqu'en 2004 au moins.

H\&M : La question du racisme dans le football s'est pourtant posée au Royaume-Uni bien avant cette période...

C. B.: Oui, mais là le problème n'était pas lié uniquement au racisme, mais plus largement à la violence autour du sport. De plus, en France on se disait que c'était un problème britannique.

À cette époque, on voulait donner une image très positive du football, le racisme était tabou et beaucoup de portes se fermaient devant nous. Si, par exemple, on venait voir une instance dirigeante, on nous renvoyait vers les services de la communication, comme on l'aurait fait avec une association de santé publique ou sociale, sans lien avec le sport. Or, nous ne voulions pas de cela, nous voulions parler avec des personnes chargées de la sécurité et de l'éducation.

H\&M : Dans le domaine de la recherche, on s'aperçoit qu'il y a beaucoup de chercheurs qui travaillent sur la question et mettent en avant le recrutement permanent de joueurs d'origine étrangère et le ratio très conséquent dans le football français. Est-ce que, à l'époque, vous vous êtes appuyés sur ces travaux? 
C. B. : Si la Licra s'est lancée dans des études, ce n'est pas parce qu'elle avait l'habitude de le faire, mais parce qu'on nous disait qu'on amplifiait le phénomène et qu'il n'y avait pas de travaux fiables là-dessus. On avait une large production sur la violence dans le football, mais rien sur les questions de racisme.

Nous avons donc mené des enquêtes. Nous avons notamment interrogé les communes. Nous avons également commandé un sondage Ipsos, ainsi qu'une étude à l'INSEP sur les problèmes de diversité dans les pôles décisionnels. Or, pour dénoncer les problèmes de racisme, il n'y avait pas d'autre voie. Les premières 
enquêtes ont permis de parler du problème. Pour le football professionnel, des événements majeurs en 2004-2008 ont conduit les médias à insister sur ce que nous dénoncions depuis un moment.

\section{H\&M : Sur les supporters, il y a peu d'études disponibles. On dénonce le problème sans l'analyser vraiment. Qu'est-ce qui a été mis en avant pour expliquer ces actes de racisme ?}

C. B.: En 2009, on a fait un sondage Ipsos avec l'Union des clubs professionnels de football (UCPF) qui est intéressant par rapport à la perception que les Français ont du racisme dans le football professionnel. Aujourd'hui, ils pensent qu'il y en a davantage, mais cela est dû à la médiatisation: je ne pense pas qu'il y ait aujourd'hui plus d'actes de racisme dans les clubs, mais leur médiatisation a beaucoup changé, donc leur perception. C'est vrai qu'on parle d'une minorité de personnes concernées, et c'est le cas, mais il ne s'agit pas d'un épiphénomène de violence : quand on va sonder des personnes intéressées par le football en leur demandant si elles ont déjà commis de ces actes de racisme, 10 \% répondent positivement. 


\section{H\&M : Quels sont les différents actes racistes rencontrés ?}

C. B. : En France, notamment, on n'a presque plus dans les stades de symboles clairs, tels que des croix gammées. Par contre, il y a des codes. Nous avons d'ailleurs réalisé un guide qui les recense, comme, par exemple, le "88" pour "Heil Hitler" ou des choses de ce type. On a des cris de singe, des bras tendus. La répression étant désormais très importante dans les stades, il y a beaucoup plus d'actes commis autour du stade, ce qui est inquiétant. Vous pouvez donc voir des personnes de couleur se faire poursuivre ou même des ratonnades. Les agressions commises en dehors du stade sont plus graves.

\section{H\&M : Le football serait-il une sorte de défouloir ?}

C. B. : Le football, ce n'est pas la guerre. Mais il y a une minorité de supporters qui pose problème. Cela est d'autant plus énervant qu'on n'arrive pas à le régler de manière systématique.

Ce qu'il faut bien comprendre, c'est que dans le football professionnel il y a des groupes organisés qui font de l'activisme politique. Il y a des militants qui viennent faire du recrutement puisqu'ils ont constaté que le sport, tout comme la musique, donnent lieu à des rassemblements qui peuvent être des réservoirs de jeunes malléables. Ils se servent de cette caisse de résonance pour faire de l'activisme politique, et c'est très organisé autour de réseaux nationaux et internationaux.

Dans le football amateur, le phénomène n'est pas aussi organisé, mais est plus la traduction exacerbée du racisme ordinaire. Nous sommes les premiers à dire que le sport peut être un outil de mixité sociale, transgénérationnel, doté de nombreux atouts positifs, mais, pour que cela sait vraiment le cas, il faut que tous les moyens soient mis en place pour limiter les forces perturbatrices, les aspects négatifs.

\section{H\&M : Par rapport à ces problèmes, qu'est-ce que la Licra a pu déve- lopper comme actions de prévention ou de lutte?}

C. B. : Par rapport aux problèmes du football professionnel, il faut agir sur le volet répressif, puisque nous avons affaire à des délinquants organisés. Pour ce qui est de la prévention, on essaye de travailler sur l'esprit critique des jeunes, pour qu'ils ne se laissent pas recruter ; on les sensibilise sur les problèmes qu'ils peuvent rencontrer et sur le fait qu'il est mieux de rejoindre certains groupes de supporters plutôt que d'autres.

Concrètement, sur la partie répressive, on a fait en sorte que le problème du racisme ne soit plus un tabou, que ce soit médiatisé afin que l'on se mobilise pour ensuite le réprimer. Il a fallu demander un arsenal législatif ; une série de lois a été votée à ce niveau-là. Par exemple, en 2000, la Licra a demandé une modification 
dans la loi sur le sport pour que les associations antiracistes puissent se constituer partie civile dans certains cas, parce que les instances sportives ne le faisaient pas. Nous avons aussi travaillé, par exemple, sur la loi visant la dissolution non seulement d'associations, mais aussi de groupements de faits, car la plupart des groupes de supporteurs ne veulent pas se constituer en associations justement pour ne pas être mis en cause. Nous avions été entendus par le ministre de l'Intérieur. Sur le plan pénal, nous avons obtenu au niveau international et national qu'il y ait un barème particulier pour les actes racistes, qu'ils soient isolés des actes de violence et reconnus à part entière, avec des sanctions individuelles pour les auteurs. Nous avons obtenu aussi que la responsabilité du club soit mise en cause, non seulement avec des sanctions financières - qui ne marchent pas -, mais aussi éventuellement avec des retraits de points, des exclusions de compétition, si le club en question ne met pas tout en ceuvre pour empêcher une partie de ses supporters de commettre de tels actes.

\section{H\&M : Est-ce que vous êtes suivis par l'ensemble du réseau associatif mobilisé contre le racisme ou est-ce qu'il y a un débat au sein de ce milieu ?}

C. B. \# Nous travaillons plus avec d'autres associations spécialisées dans le milieu du sport. On travaille notamment avec des associations qui luttent contre l'homophobie, le trafic de jeunes footballeurs sur les questions d'immigration, d'autres encore qui travaillent sur l'éducation par le sport. Nous travaillons en réseau avec des associations spécialisées dans ce domaine à la fois dans le milieu français et dans le milieu international. Étant donné que nos "ennemis" travaillent au niveau international, il est important que nous puissions en faire de même. Nous faisons ainsi partie du bureau de FARE, Football Against Racism in Europe.

\section{H\&M : Ce réseau est-il soutenu par l'Union européenne ?}

C. B.: Il est soutenu surtout par l'UEFA. Il y aussi des financements de la Commission européenne sous forme d'un appel à projets.

\section{H\&M : Est-ce qu’on peut établir un panorama européen, avec des pays qui seraient très performants en la matière et pourraient donc servir d'exemple?}

C. B. Il y a le modèle britannique, dont on parle beaucoup, mais le problème dans ce cas est qu'il y a moins de racisme dans les stades dans la mesure où les personnes issues des milieux populaires sont moins nombreuses à y venir. Le prix des places a tellement augmenté qu'une sélection s'est opérée par le niveau de revenu, ce qui fait 
que la composition sociologique dans les stades n'est plus la même. Or, il faut comprendre que, dans la plupart des pays, la situation du football n'est pas forcément comparable. En France, il n'y a pas le même engouement pour le football : Lens, Marseille et Saint-Étienne sont des exceptions. À Londres, il y a six clubs professionnels, tandis qu'à Paris on imagine mal un deuxième club professionnel. Ce n'est pas le cas en Europe : à Barcelone et Madrid il y a deux clubs par ville, même chose à Munich, Rome ou Milan. Si vous prenez les pays du sud de l'Europe, comme l'Espagne ou l'Italie, ou les pays de l'Est, les problèmes qu'on y rencontre sont sans commune mesure avec ceux qu'on trouve en France. Ils y sont beaucoup plus graves, il y a beaucoup plus de racisme et de violence. De même, il y a de gros problèmes de nationalisme en l'Europe de l'Est, ce qui est très inquiétant par rapport à l'Euro qui va se tenir en 2012 en Pologne et en Ukraine.

\section{H\&M : Est-ce que cela reflète un état de la société nationale ?}

C. B. : Oui. En Italie, par exemple, on n'arrive pas à accepter Mario Balotelli, qui est un joueur italien noir, âgé de dix-neuf ans, de l'Inter de Milan. Une grande partie de la population italienne ne le veut pas dans l'équipe nationale, ce qui va à l'encontre des intérêts de l'équipe. Ils préfèrent ne pas avoir un Noir et il subit fréquemment des propos racistes. 
H\&M : Pouvez-vous évoquer le phénomène du recrutement des jeunes joueurs étrangers, et notamment africains? Quelles sont vos actions dans ce domaine? Peut-on parler de trafic, avec parfois des destins tragiques pour ces jeunes?

C. B. : Notre but est de faire sortir les questions taboues, mais nous ne sommes pas une association spécialisée dans les questions de droit d'asile ou autre. Nous travaillons donc avec d'autres organismes spécialisés dans ce domaine. On a réussi à faire émerger la question et on aimerait maintenant travailler sur le volet prévention, en Afrique, afin de sensibiliser les équipes et les jeunes par rapport à ces phénomènes, mais on n'arrive même pas à avoir de chiffres précis de la part des associations.

\section{H\&M : Êtes-vous plutôt alertés sur des situations personnelles ?}

C. B. : Nous sommes alertés, mais, lorsque nous demandons à ces associations des dossiers ou des informations, nous avons de plus en plus de mal à en avoir, donc c'est quelque chose de très compliqué à quantifier. Néanmoins, il est très important de pouvoir travailler sur les pays d'origine.

\section{H\&M : L'idée n'est pas de limiter des transferts de compétences venant de l'étranger, qui ont toujours existé et qui font la vitalité du football en France, mais de mieux organiser ce phénomène, d'assurer les droits de ces jeunes.}

C. B. : Oui, le problème est que les jeunes amenés sur le territoire sont engagés avec des contrats sans véritable valeur juridique. Lorsqu'ils se blessent ou que leur niveau baisse, ces mineurs se retrouvent sans contrat, sans logement, sans papiers. C'est là un problème qui relève du respect des droits de l'homme. Si le jeune en question est protégé, son transfert ne pose pas de problème en soi, mais il doit être réalisé dans les règles de l'art.

Parfois, on compare cette pratique à celle des jeunes Français recrutés par les clubs anglais. Or, cela n'est pas du tout la même chose à mon avis. Dans ce cas, le public est suffisamment alerté, nous ne sommes pas du tout confrontés aux mêmes problèmes de droits de l'homme que ceux induits par ces migrations Sud-Nord. 